Check for updates

Cite this: Phys. Chem. Chem. Phys., 2019, 21, 13040

Received 11th March 2019, Accepted 30th May 2019 DOI: $10.1039 / c 9 c p 01387 c$ rsc.li/pccp

\section{Cation diffusion patterns across the magneto-structural transition in $\mathrm{Fe}_{7} \mathrm{~S}_{8}$}

\author{
Dimitrios Koulialias, ${ }^{a b}$ Peter G. Weidler, ${ }^{c}$ Michalis Charilaou, ${ }^{d}$ Jörg F. Löffler ${ }^{b}$ and \\ Andreas U. Gehring (iD *a
}

\begin{abstract}
Migration of atoms in solids during diffusion-dependent reactions is relatively fast and generally not directly recordable in experiments. Here we present an experimental framework that includes fast differential scanning calorimetry to resolve cation-migration paths in crystalline solids using the reversible magneto-structural transition of $4 \mathrm{C}$ to $1 \mathrm{C}$ pyrrhotite as a testbed. The transition between these two polymorphic $\mathrm{Fe}_{7} \mathrm{~S}_{8}$ phases at about $600 \mathrm{~K}$ is a diffusive process of vacancies, respectively of Fe in octahedral interstitial sites within a hexagonal close-packed lattice of sulfur, and it coincides with the Curie temperature of $4 \mathrm{C}$ pyrrhotite. The Fe cations migrate along three kinds of diffusion paths, and their enthalpy contributions to the total reaction enthalpy are taken to define the diffusion patterns in the endothermic reaction and the exothermic back-reaction, respectively. Our experimental findings provide insight into the potential of diffusion patterns to disentangle ordering mechanisms in solids.
\end{abstract}

\section{Introduction}

Metal-deficient solid phases, mainly oxides $\left(\mathrm{M}_{1-x} \mathrm{O}\right)$ or sulfides $\left(\mathrm{M}_{1-x} \mathrm{~S}\right)$, exhibit physical properties that are of broad interest in Earth and astrophysical sciences to constrain physico-chemical processes during planetary evolution, but also in materials science as a concept to design functional compounds. ${ }^{1-6}$ These defective phases have in common that they undergo structural transitions under changing temperature and/or pressure conditions due to rearrangement of the cation-deficient sites. ${ }^{7-10}$

Among defective solids the minerals of the pyrrhotite group $\left(\mathrm{Fe}_{1-x} \mathrm{~S} ; 0<x \leq 0.125\right)$ represent a classic omission solidsolution series formed by a hexagonal close-packing of sulfur with ordered Fe sites and vacancies in the octahedral interstices that results in a hexagonal NiAs-type substructure and variable commensurate and incommensurate superstructures. ${ }^{11,12}$ Slight changes in the chemical composition and the subsequent arrangement of the empty sites generate a rich variation of electromagnetic states and processes. ${ }^{11,13-15}$ At temperatures of about $600 \mathrm{~K}$ the superstructures of all pyrrhotite phases are unstable due to diffusion-controlled processes that randomize the Fe-deficient sites in a hexagonal $1 \mathrm{C}$ structure. ${ }^{9,16,17}$ Because of the unique

\footnotetext{
${ }^{a}$ Institute of Geophysics, ETH Zurich, 8092 Zurich, Switzerland. E-mail: agehring@ethz.ch

${ }^{b}$ Laboratory of Metal Physics and Technology, Department of Materials, ETH Zurich, 8093 Zurich, Switzerland

${ }^{c}$ Institute of Functional Interfaces, Karlsruhe Institute of Technology, 76131 Karlsruhe, Germany

${ }^{d}$ Department of Physics, University of Louisiana at Lafayette, Lafayette, LA 70504, USA
}

structural and physico-chemical properties of the members of the pyrrhotite group and their potential for technological applications, great effort has been put into the synthesis of pyrrhotite nanostructures such as nanotubes and nanowires., ${ }^{5,-21}$ The inherent difficulty associated with pyrrhotite is the synthesis of structurally and magnetically well-defined omission phases with highly ordered and stable arrangements of empty sites. Therefore, apart from a fundamental physio-chemical interest, detailed information on the cation-diffusion processes is a requirement on the route to the technological application of the pyrrhotite phases.

The Fe-deficient end-member (ideal formula $\mathrm{Fe}_{7} \mathrm{~S}_{8}$ ) of the pyrrhotite omission series, denoted $4 \mathrm{C}$ pyrrhotite, is the ideal testbed to study diffusion mechanisms. The $4 \mathrm{C}$ phase has a monoclinic superstructure that stems from highly ordered vacancies in a stacking sequence of full and vacancy-bearing layers with a four-fold modulation per unit cell. Bertaut ${ }^{22}$ suggested that this ordering is a result of the Coulomb repulsion minimization of the Fe-deficient sites. The vacancy order generates a relatively strong ferrimagnetism (magnetic moment $m \approx 2.73 \mu_{\mathrm{B}}$ per Fe atom $^{23}$ ) that stems from magnetically uncompensated sublattices due to ferromagnetic intra-layer and antiferromagnetic inter-layer couplings of the Fe magnetic moments with a Curie temperature $\left(T_{\mathrm{C}}\right)$ of about $600 \mathrm{~K} \cdot{ }^{24,25}$ The loss of spontaneous magnetization occurs in the same temperature range as the polymorphic transition of the monoclinic 4C into a hexagonal 1C pyrrhotite. This transition goes along with a vacancy rearrangement that can be considered as a reversible diffusive process (e.g., ref. 26-28). In the 4C structure one of the four different $\mathrm{Fe}^{2+}$ sites has an occupancy probability of 0.5 and for the others it is zero, whereas in $1 \mathrm{C}$ the occupancy probability is 


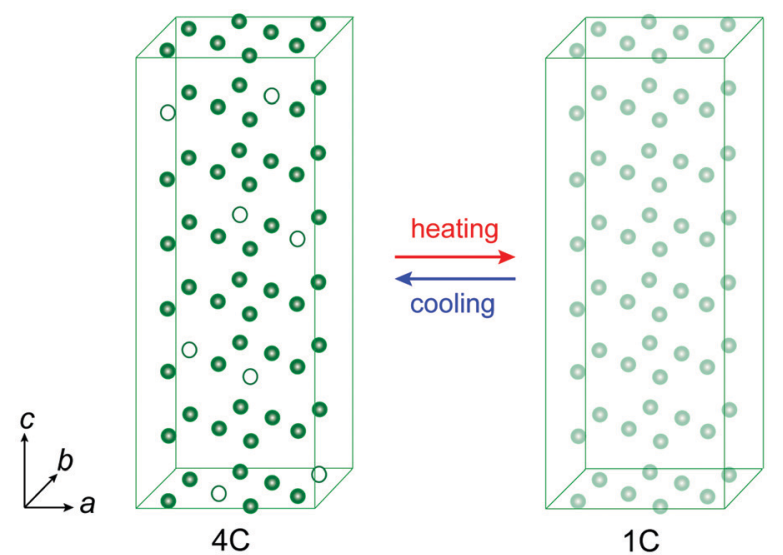

Fig. 1 Schematic delineation of the cation arrangements in the polymorphous $4 \mathrm{C}$ and $1 \mathrm{C}$ structures with Fe sites having occupancy probabilities of 1 (green dots), 0 (circles) and 0.875 (light green dots), respectively. The sulfur atoms are omitted for clarity reason. Heating above $T_{\mathrm{C}} \approx 600 \mathrm{~K}$ generates the $4 \mathrm{C}$ to $1 \mathrm{C}$ transition, while the back-reaction occurs upon cooling.

theoretically 0.875 per $\mathrm{Fe}^{2+}$ site (Fig. 1). In the $4 \mathrm{C}$ pyrrhotite, the $\mathrm{Fe}^{2+}$ sites can schematically be subdivided into three crystallographic positions with inter-layer, intra-layer, or no vacancies in their vicinity and hence the cations have different diffusion coefficients and myriad combinations for their migration. Radiotracer-diffusion experiments and numerical simulations showed that inter-layer dominates intra-layer cation migrations. ${ }^{29,30}$ This information on the self-diffusion in pyrrhotite suggests anisotropy effects but provides only limited insight into the mechanism behind the reversible $4 \mathrm{C}$ to $1 \mathrm{C}$ transition, because in pyrrhotite the intrinsic diffusion is mainly determined by competing jumps of the Fe cations in altering structural configurations caused by vacancies. This complexity is the main reason why intrinsic diffusion processes in pyrrhotite and generally in defective structures are unresolved. Here, we present an experimental framework that opens a new way to decipher diffusion patterns in a methodological approach that can be extended towards a wide range of defective solids.

\section{Materials and methods}

In this study a natural 4C pyrrhotite crystal from the locality of Morro Velho (Minas Gerais, Brazil) was chosen. ${ }^{23}$ For the detailed crystallographic, chemical, and magnetic analyses powder samples were prepared by crushing and subsequently grinding the crystal sample in an agate mortar for about 30 minutes. For the calorimetric measurements crystal flakes were used. Powder X-ray diffractometry (XRD) at room temperature using untreated and heated samples was performed with a Bruker D8 device with $\mathrm{Cu}$ $\mathrm{K}_{\alpha 1,2}$ radiation, and the patterns were Rietveld-refined with the Bruker software TOPAS. ${ }^{23,31}$ The chemical composition was determined by energy-dispersive X-ray fluorescence (ED-XRF) on a SPECTRO XEPOS spectrometer. The magnetic susceptibility upon heating between 300 and $650 \mathrm{~K}$ was determined on an AGICO KLY2 Kappabridge in argon atmosphere with a heating/cooling rate of about $10 \mathrm{~K} \mathrm{~min}^{-1}$.
Complementary thermal analysis of the magneto-structural transition was performed by differential scanning calorimetry (DSC) on crystal flakes (with masses between 10.5 and $18 \mathrm{mg}$ ) at heating/cooling rates between 1 and $30 \mathrm{~K} \mathrm{~min}^{-1}$ in a MettlerToledo DSC 1 device. For more detailed insight into the mechanism of the transition fast differential scanning calorimetry (FDSC) measurements were performed on microflakes at heating/cooling rates between 10 and $200 \mathrm{~K} \mathrm{~s}^{-1}$ using a MettlerToledo Flash DSC 1 with UFS1 sensor. The sensor support temperature was selected to $183 \mathrm{~K}$ and argon was used for purging. According to ref. 32 a difference in the temperature scale of $8 \mathrm{~K}$ was determined between FDSC and DSC. The FDSC temperatures were calibrated using an indium standard sample. The onsets of the endo- and exothermic events were determined after background correction using the Mettler-Toledo STARe software (Version V16.10). In the FDSC experiment, the heating/ cooling cycles were continuously recorded with decreasing rate.

\section{Results and discussion}

\subsection{Structural and magnetic properties}

The powder XRD pattern of the Morro Velho sample shows a 4C pyrrhotite and no impurity phase was detected (Fig. 2, inset). The XRF analysis shows a chemical composition of $46.17 \pm$ 0.1 at $\%$ Fe and $53.83 \pm 0.1$ at $\%$ S, and 377 ppm Ni traces, which corresponds to $\mathrm{Fe}_{6.9} \mathrm{~S}_{8}$. The $4 \mathrm{C}$ pyrrhotite XRD pattern can be well refined in the $C 2 / c$ space group with the structural model by Powell et $a l^{27}$ The unit-cell parameters obtained at room temperature were $a=1.1918(2) \mathrm{nm}, b=0.6872(2) \mathrm{nm}, c=$ $1.2913(2) \mathrm{nm}$, and $\beta=118.035(1)^{\circ}$ with a goodness-of-fit of 3.67 , a weighted Durbin-Watson value of 0.24 , and an $R$-Bragg factor of 5.57. The refinement of the Fe-occupancy factor yielded a chemical composition of $\mathrm{Fe}_{6.6} \mathrm{~S}_{8}$ that is slightly

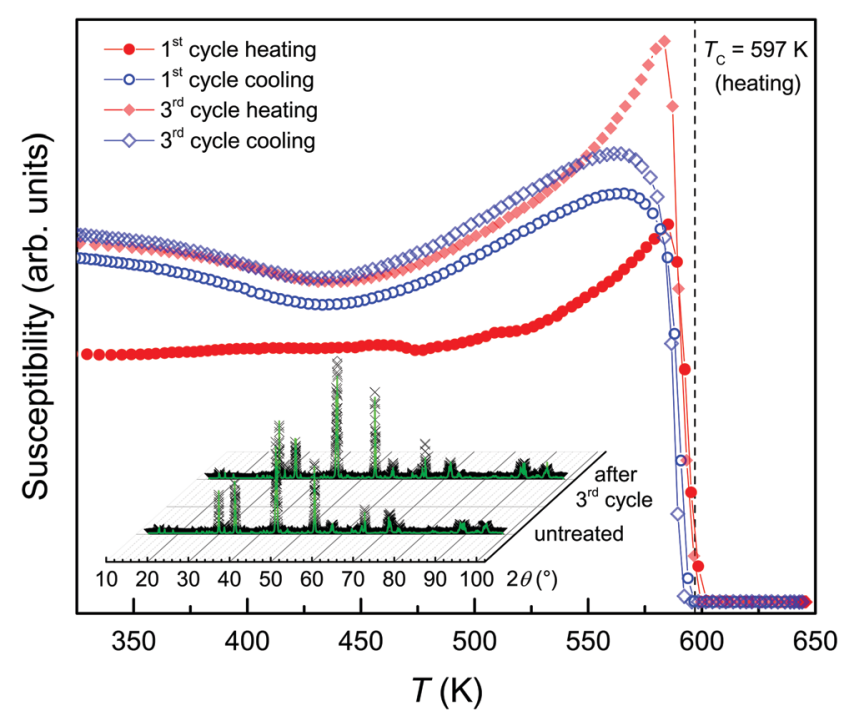

Fig. 2 Magnetic susceptibility of the pyrrhotite powder during heating (red) and cooling (blue) cycles. Inset: XRD patterns of the untreated sample and after three heating/cooling cycles. The Curie temperature $\left(T_{C}\right)$ upon heating is marked (dashed line). 
Fe-depleted compared to the $\mathrm{Fe}_{6.9} \mathrm{~S}_{8}$ obtained from the chemical analysis. A mean crystallite size of about $220 \mathrm{~nm}$ was determined for the powder using the Scherrer equation ${ }^{33}$ after considering the instrumental and stress/strain contributions to the line broadening.

The magnetic susceptibility $(\chi)$ of the heating-cooling cycles of the 4C pyrrhotite is shown in Fig. 2 for temperatures between 320 and $625 \mathrm{~K}$. During the first heating, $\chi$ is constant up to $450 \mathrm{~K}$, followed by an increase with a peak at $584 \mathrm{~K}$ and a drop down towards $600 \mathrm{~K}$. The peak can be attributed to the Hopkinson effect, which is commonly observed in ferro-/ferrimagnetic materials just before the Curie temperature. ${ }^{34}$ For multi-domain particles, it is explained by enhanced domain-wall mobility with increasing temperature, which leads to an increase in the magnetic permeability and in turn to an increase in $\chi$ (e.g., ref. 35). Using the tangent method, ${ }^{36}$ a Curie temperature $T_{\mathrm{C}}=597 \mathrm{~K}$ was determined that is consistent with the values for $4 \mathrm{C}$ pyrrhotite reported in the literature (e.g. ref. 25 and 27). Troilite, the vacancyfree end-member of the pyrrhotite omission series has a Néel temperature $T_{\mathrm{N}} \approx 600 \mathrm{~K}$ (e.g. ref. 37 and 38), and therefore it is feasible to assume that the loss of magnetic ordering in the $4 \mathrm{C}$ phase is not critically affected by the vacancies. In the cooling curve, the onset of $\chi$, however, occurs at $594 \mathrm{~K}$, which indicates a minor hysteretic effect of $3 \mathrm{~K}$ between the heating and cooling curves. Upon further cooling, $\chi$ steeply increases down to $580 \mathrm{~K}$, and reaches a maximum at $565 \mathrm{~K}$ followed by a minor overall decrease with a broad local minimum at $435 \mathrm{~K}$. At $300 \mathrm{~K}$, after the first heating/cooling cycle, $\chi$ is slightly higher compared to the initial value. In the subsequent heating curves, the Hopkinson peak becomes more pronounced but its peak position remains unchanged. The Curie temperatures and the hysteretic shifts between heating and cooling curves also remain constant within the experimental error. The cooling curves are nearly identical, but the comparison of the heating/cooling cycles indicates minor magneto-structural modifications during the first heating and this establishes a new thermodynamic equilibrium in the sample.

The XRD pattern obtained from the powder sample after the three cycles shows a single-phase $4 \mathrm{C}$ pyrrhotite (Fig. 2, inset) with the refined unit-cell parameters $a=1.1914(3) \mathrm{nm}, b=$ $0.6870(2) \mathrm{nm}, c=1.2911(3) \mathrm{nm}$, and $\beta=118.025(1)^{\circ}$ with a goodness-of-fit of 3.96 , a weighted Durbin-Watson value of 0.21 , and a $R$-Bragg factor of 6.73 . The comparison of the crystallographic parameters of the untreated and the heat-treated samples reveals no significant changes. The chemical composition of $\mathrm{Fe}_{6.9} \mathrm{~S}_{8}$ in the heated sample obtained from the refinement of the Fe-occupancy factor is equal to that yielded from the XRF analysis. Simultaneous to the shift in stoichiometry due to the heat treatment, the mean crystallite size in the powder increases by about 50\% to $330 \mathrm{~nm}$. The heat-induced sintering can also explain the more pronounced Hopkinson peaks, because larger crystallites in pyrrhotite grains are expected to contain more magnetic domain walls. The sintering can also account for the shift towards the ideal 4C stoichiometry in the cycled sample because small defects, such as stacking faults forming jogs, ${ }^{1}$ which are not detectable by XRD, can be eliminated by the heat treatment. Under the assumption that these defects are less Fe-deficient compared to the bulk material, their healing can explain the shift towards a stoichiometric pyrrhotite. Taking the susceptibility data into account, it is obvious that the sintering has no critical effect on the magnetic ordering in the $4 \mathrm{C}$ phase.

\subsection{Calorimetric properties}

Thermodynamic analysis of the magneto-structural transition using a scanning rate of $10 \mathrm{~K} \mathrm{~min}^{-1}$ for the DSC cycles shows no apparent changes for temperatures between $300 \mathrm{~K}$ and $575 \mathrm{~K}$ (Fig. 3). Above $575 \mathrm{~K}$, the three DSC cycles reveal an onset of the endothermic event at $595.4 \pm 0.4 \mathrm{~K}$ upon heating with a mean peak temperature of $600.5 \pm 0.4 \mathrm{~K}$. For the exothermic event upon cooling the onset is at $593.4 \pm 0.8 \mathrm{~K}$ with a mean peak temperature of $586.8 \pm 0.2 \mathrm{~K}$. The onsets of both events are slightly below $T_{\mathrm{C}}=597 \mathrm{~K}$ of $4 \mathrm{C}$ pyrrhotite, which accords with previous experimental studies. ${ }^{25,39}$

For the transition of $4 \mathrm{C}$ to $1 \mathrm{C}$ pyrrhotite, the enthalpy changes $\Delta H_{4 \mathrm{C} \rightarrow 1 \mathrm{C}}$ are $0.77 \mathrm{~kJ} \mathrm{~mol}^{-1}$ in the first two cycles and slightly increase to $0.81 \mathrm{~kJ} \mathrm{~mol}^{-1}$ in the third one. These $\Delta H_{4 \mathrm{C} \rightarrow 1 \mathrm{C}}$ values are similar to those of a natural $4 \mathrm{C}$ pyrrhotite crystal from a different locality ${ }^{1}$ and somewhat lower than the $1.3 \mathrm{~kJ} \mathrm{~mol}^{-1}$ obtained from a synthetic $4 \mathrm{C}$ powder sample. ${ }^{28}$ Considering the $\mathrm{XRD}$ data, the increase in $\Delta H_{4 \mathrm{C} \rightarrow 1 \mathrm{C}}$ during the cycling is probably a sintering effect, because more energy is needed for the $4 \mathrm{C}$ to $1 \mathrm{C}$ transition to occur when the vacancy order gets enhanced. The sintering also slightly changes the shape of the endothermic peak. In the third cycle, it is weakly split and can be fitted with two Gaussian components, and this in turn points to two events that slightly differ in their $\Delta H_{4 \mathrm{C} \rightarrow 1 \mathrm{C}}$ (Fig. 3, inset). Taking this into account, the splitting provides evidence that the breakdown of the vacancy order in $4 \mathrm{C}$ pyrrhotite is subject to a heterogeneous diffusion mechanism, i.e., the probability for a cation movement is unequal for the four Fe-sites in the $4 \mathrm{C}$ pyrrhotite. Regarding the exothermic event the DSC curves exhibit asymmetric shapes that can only be fitted by three Gaussian components.

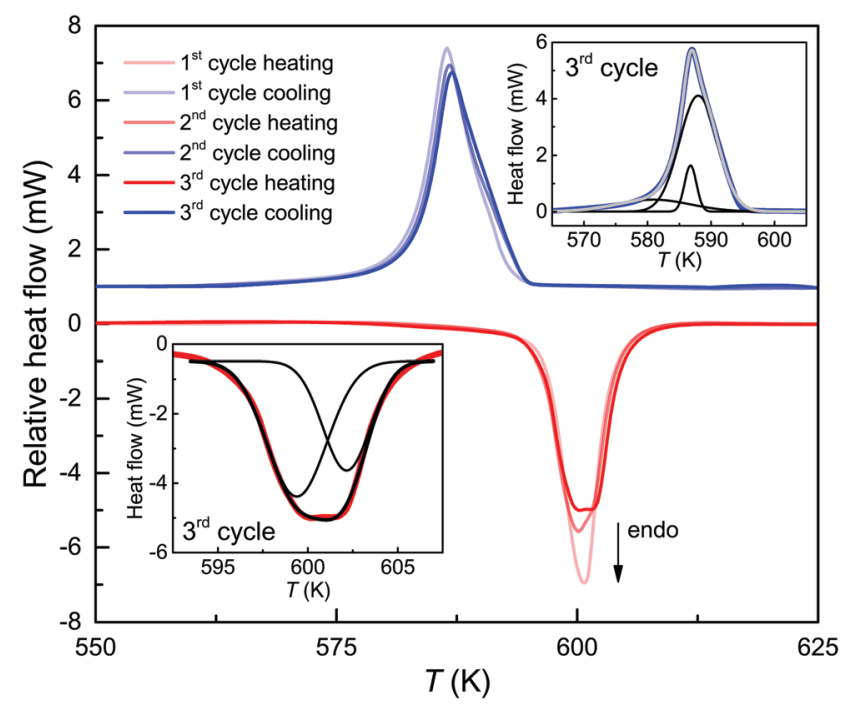

Fig. 3 Differential scanning calorimetry of a pyrrhotite flake with three heating (red) and cooling (blue) scans. Insets: Curve fitting of the endo- and exothermic events of the third scan into two, respectively three Gaussian components and their corresponding sums. 


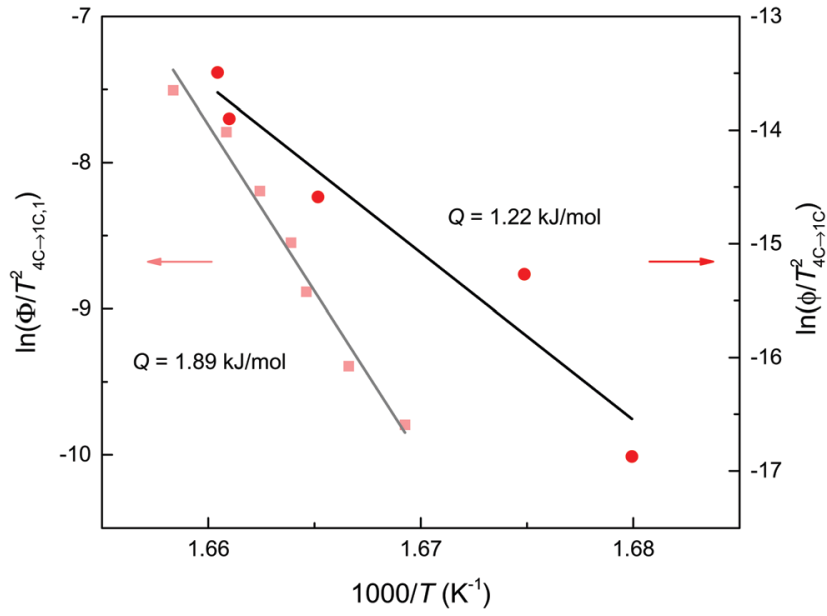

Fig. 4 Kissinger plots obtained from the DSC measurements (full circles) and from first peaks in the FDSC measurements (squares) with their corresponding activation energies $Q$. The error bars are smaller than the symbol size.

This indicates that the $1 \mathrm{C}$ to $4 \mathrm{C}$ transition is also a heterogeneous diffusion process (Fig. 3). The enthalpy changes $\Delta H_{1 \mathrm{C} \rightarrow 4 \mathrm{C}}$ obtained from the three cycles differ only slightly and their mean is $-0.93 \pm 0.03 \mathrm{~kJ} \mathrm{~mol}^{-1}$.

The change of the scanning rates in the DSC cycles has only minor effects on the onset temperatures with means of $595.1 \pm$ $1.3 \mathrm{~K}$ for the endothermic and $592.9 \pm 0.8 \mathrm{~K}$ for the exothermic event. The enthalpy changes are also nearly rate-independent with means $\Delta H_{4 \mathrm{C} \rightarrow 1 \mathrm{C}}=0.82 \pm 0.05 \mathrm{~kJ} \mathrm{~mol}^{-1}$ and $\Delta H_{1 \mathrm{C} \rightarrow 4 \mathrm{C}}=-0.81 \pm$ $0.10 \mathrm{~kJ} \mathrm{~mol}^{-1}$, respectively (Fig. 3). In contrast, the difference of the heating and cooling peaks reveals a non-linear increase with the rate from $5 \mathrm{~K}$ at $1 \mathrm{~K} \mathrm{~min}^{-1}$ to $21 \mathrm{~K}$ at $30 \mathrm{~K} \mathrm{~min}^{-1}$ with a symmetric shift at around $593 \mathrm{~K}$. Furthermore, taking the mean of the peak temperature $T_{\text {peak }}$ of the three scans for each heating rate, the apparent activation energy $Q$ for the endothermic reaction can be extracted using the Kissinger relation $\ln \left(\Phi / T_{\text {peak }}{ }^{2}\right) \propto 1 / T_{\text {peak }}$, where $\Phi$ is the heating rate (Fig. 4). From the slope of the Kissinger plot $m$, the activation energy $Q=m R$ can be determined (e.g. ref. 40), where $R$ is the universal gas constant. A value of $Q=1.22 \mathrm{~kJ} \mathrm{~mol}^{-1}$ is obtained. The relative large scatter of the peak temperature data in Fig. 4 is probably caused by the influence of the heat transfer change of the sample due to sintering. The energy barrier, however, is nearly two orders of magnitudes lower than that obtained from radiotracer experiments. ${ }^{16,29}$ In such experiments, the tracer migration is a relatively slow process, and therefore it can mask the faster intrinsic diffusion. Moreover, Herbert et al. ${ }^{16}$ provided evidence that the increase of the relative, spontaneous magnetization $(M)$ expressed as $M(T) / M(T=0 \mathrm{~K})$ enhances the $Q$ of the selfdiffusion. Considering the magnetic data for $4 \mathrm{C}$ pyrrhotite reported by Powell et al., ${ }^{27} M$ is nearly zero in the temperature range of the structural transition. Therefore, a critical, magnetic effect on the intrinsic diffusion is unlikely.

\subsection{Diffusion patterns}

In order to provide further insight into the mechanism behind the diffusion-controlled reaction, FDSC is applied. In contrast
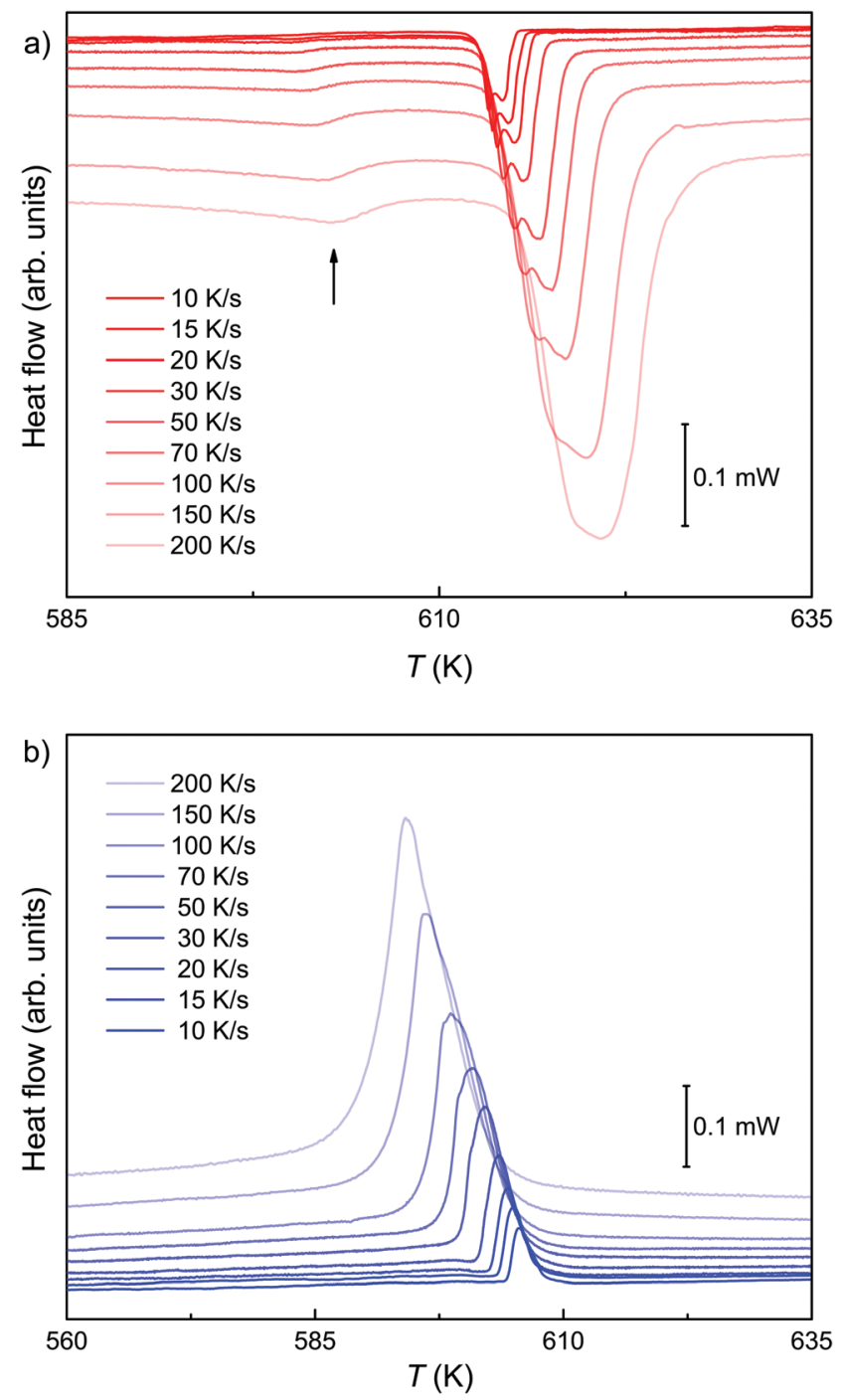

Fig. 5 FDSC of a pyrrhotite microflake recorded with rates between 10 and $200 \mathrm{~K} \mathrm{~s}^{-1}$ during heating (a) and (b) cooling. The first events in the heating curves are marked by an arrow.

to DSC, the peak positions and the shapes of the endothermic event are congruent in the FDSC scans with the same heating rate. The FDSC curves with heating rates between 10 and $100 \mathrm{~K} \mathrm{~s}^{-1}$ separate the endothermic event into three peaks, a relatively broad one and a doublet at relatively higher temperature (Fig. 5a). At higher rates up to $200 \mathrm{~K} \mathrm{~s}^{-1}$, two features of the doublet blur (Fig. 5a and b). The asymmetric peaks in the cooling curves have similar shapes as in the conventional DSC measurements. (Fig. $3 \mathrm{~b}$ and $5 \mathrm{~b}$ ).

Assuming that the total transformation enthalpies $\left(\Delta H_{\text {tot }}\right)$ obtained from DSC correspond to those from FDSC, the enthalpies of the $4 \mathrm{C}$ to $1 \mathrm{C}$ and the $1 \mathrm{C}$ to $4 \mathrm{C}$ transformations can both be subdivided into three contributions $\Delta H_{i}(i=1,2,3)$ derived from the three Gaussian components (Fig. 6) with $\Delta H_{\mathrm{tot}}=\sum_{i=1} \Delta H_{i}$. The relative contributions are then $\Delta H_{i} / \Delta H_{\text {tot }}$.

The $\Delta H_{i}$ is affected by the three crystallographic positions of $\mathrm{Fe}$ cations with respect to the vacancy sites, i.e., by the diffusion 

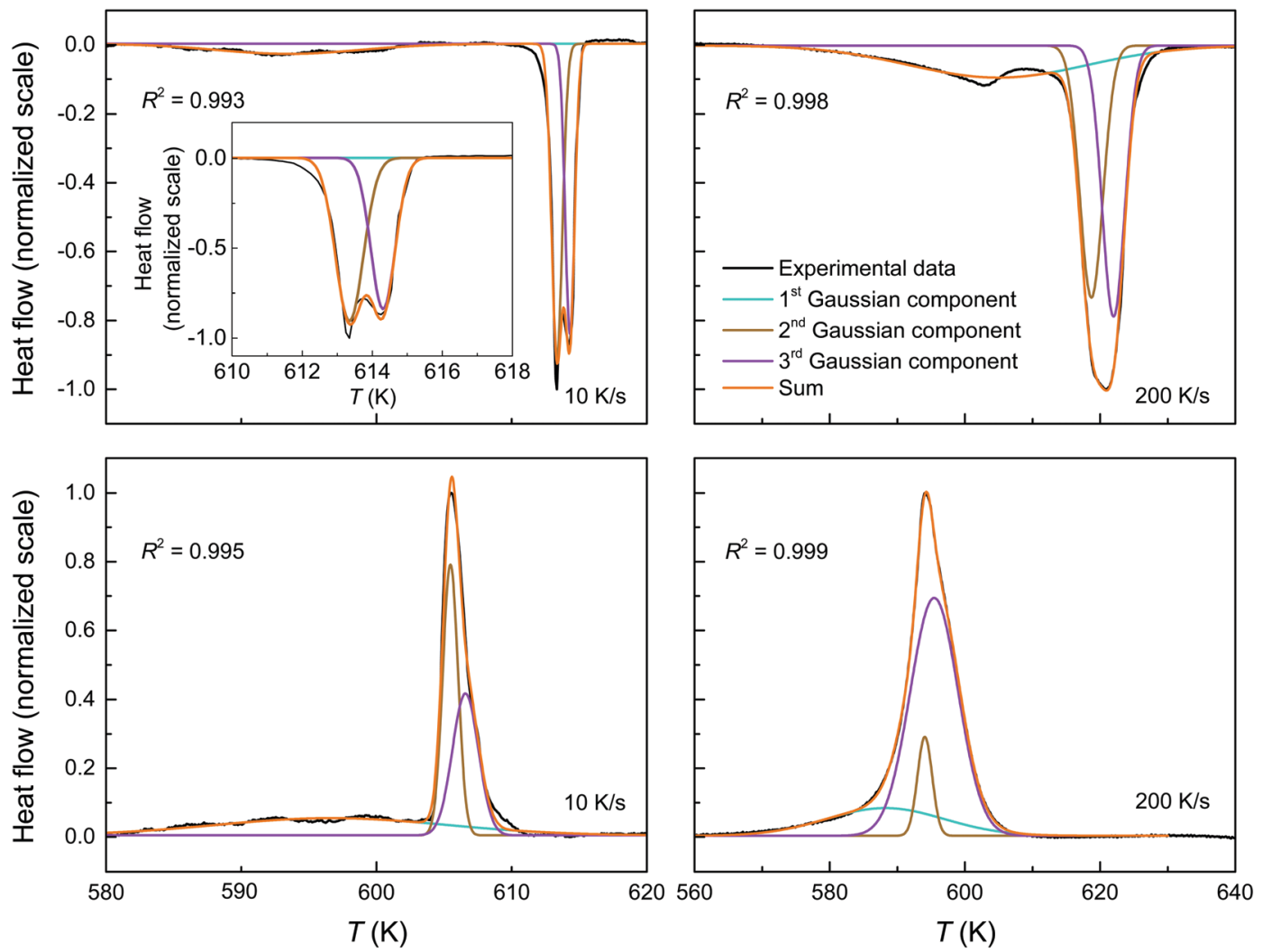

Fig. 6 Splitting of the endothermic (upper panels) and exothermic (lower panels) FDSC curves recorded at rates of 10 and $200 \mathrm{~K} \mathrm{~s}^{-1}$ into three Gaussian components. The fit accuracy is given by the $R$-square value.

coefficient and the diffusion path (Fig. 1). Considering the hexagonal close-packing of the sulfur atoms in the pyrrhotite structure, three kinds of paths for the $\mathrm{Fe}^{2+}$ cations can be inferred. There is a direct diffusion jump into a vacancy via the openings formed by face-shared sulfur octahedra in the $c$-plane with only one degree of freedom along the $c$-axis $(\mathrm{o}-\mathrm{O})$ (Fig. 7). In the 4C structure with the stacking sequence of full and vacancy layers, one quarter of the Fe sites can follow such path (Fig. 1). For the other Fe sites the cations may migrate through tetrahedral interstices either in the $c$-plane $(\mathrm{o}-\mathrm{t}-\mathrm{o})$ or along the $c$-axis (o-t-t-o) with degrees of freedom $>1$ (Fig. 7). The different paths vary by their lengths. The shortest one with nearly $0.30 \mathrm{~nm}$ is $\mathrm{o}-\mathrm{O}$ followed by $\mathrm{o}-\mathrm{t}-\mathrm{o}$ and $\mathrm{o}-\mathrm{t}-\mathrm{t}-\mathrm{O}$ with lengths of about $0.37 \mathrm{~nm}$ and $0.55 \mathrm{~nm}$, respectively (Fig. 7). It was shown that self-diffusion along the $c$-axis is faster than in the $c$-plane.$^{29}$ Powell et al. ${ }^{27}$ also demonstrated that with the onset of the transition the occupancy probability of Fe sites next to vacancies decreases from 0.5 to 0.38 . Thus, it can be assumed that the Fe migrations in $4 \mathrm{C}$ pyrrhotite starts along the o-o path, which is energetically more favorable compared to those via tetrahedral interstices. With this in mind the initial o-o jumps can be attributed to $\Delta H_{1}$. They break the local cation-vacancy relations in the $4 \mathrm{C}$ structure while the number of vacancies remains constant. These $\mathrm{o}-\mathrm{o}$ jumps are thus expected to trigger a state with a dynamic opening and closing of diffusion paths, where all the cations are captured and encountered until a new equilibrium in the Fe-vacancy configuration

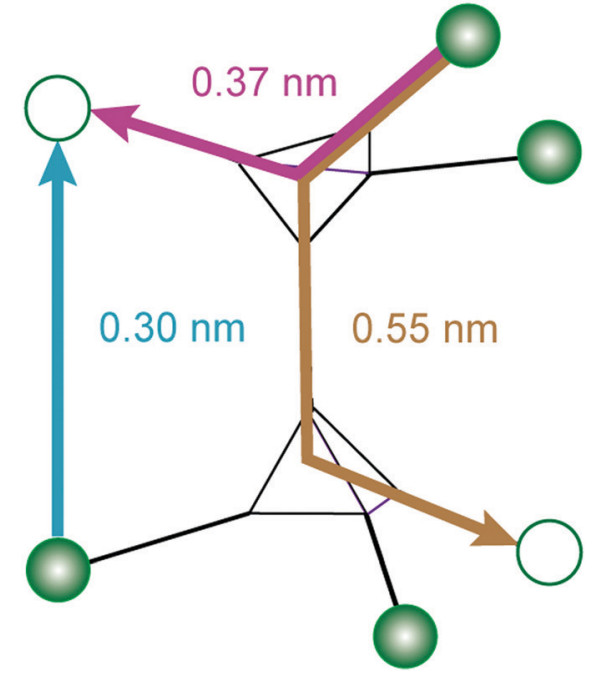

Fig. 7 Schematic display of three kinds of cation-diffusion paths in the NiAs-type structure with direct jumps along the c-direction (o-O) and intra- and inter-layer jumps via tetrahedral interstices, $(\mathrm{o}-\mathrm{t}-\mathrm{O})$ and $(\mathrm{o}-\mathrm{t}-$ $\mathrm{t}-\mathrm{O})$, respectively.

is established. In the 1C structure this configuration shows no crystallographic long-range order. At $T<600 \mathrm{~K}$, the initial o-O jumps $\left(\Delta H_{1}\right)$, however, are always unaffected by the two hightemperature events $\Delta H_{2}$ and $\Delta H_{3}$, and, therefore, they can be taken to determine the activation energy of the initial diffusion 

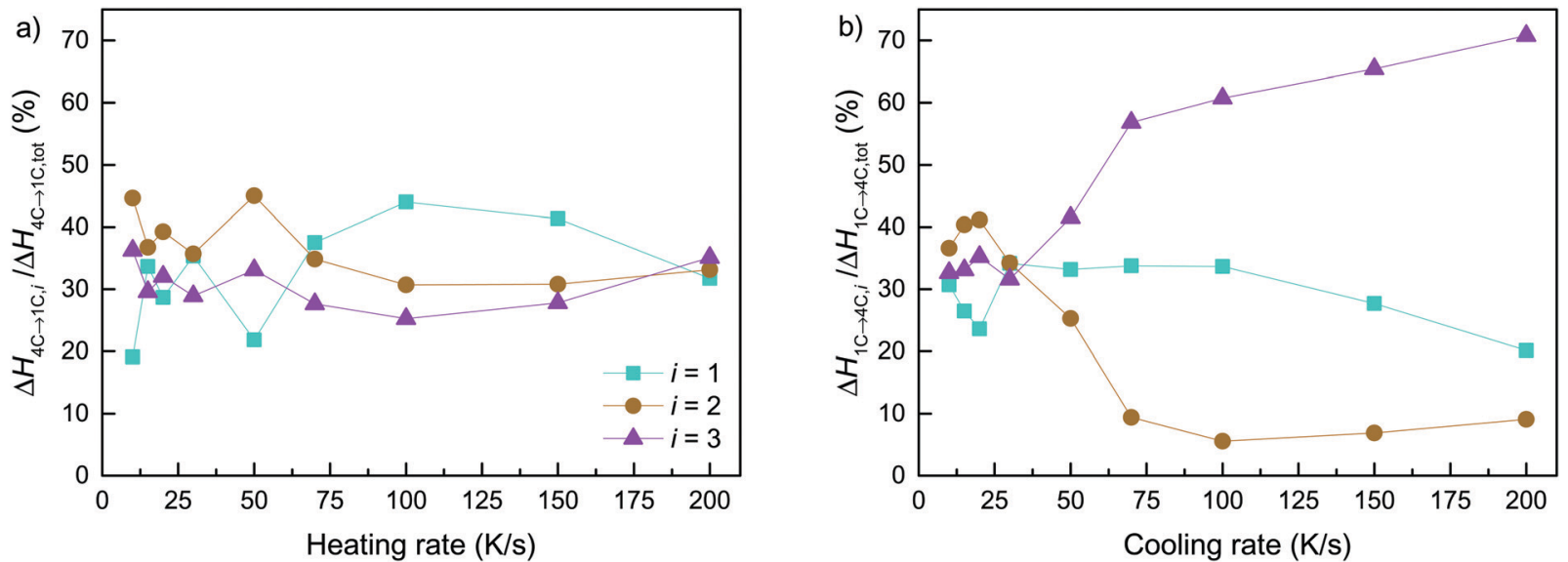

Fig. 8 Rate-dependence of the relative enthalpy contributions for the three different diffusion paths with respect to the endothermic (a) and exothermic (b) reactions.

step using the Kissinger plot (Fig. 4). The yielded activation energy of $Q=1.89 \mathrm{~kJ} \mathrm{~mol}^{-1}$ is similar to that of the overall diffusion obtained from the average peak $T_{\text {peak }}$ of the conventional DSC measurements. This is consistent with the idea that the initial $\mathrm{o}-\mathrm{O}$ jumps destabilize the $4 \mathrm{C}$ structure and thus trigger the breakdown of the cation order in which all sites become involved. With this in mind the enthalpy contributions $\Delta H_{2}$ and $\Delta H_{3}$ indicate the onset of the diffusion along the o-t-o and $\mathrm{o}-\mathrm{t}-\mathrm{t}-\mathrm{o}$ paths, respectively. In fact, the diffusion patterns obtained from the FDSC curves with variable heating rates are described by three components $\Delta H_{4 \mathrm{C} \rightarrow 1 \mathrm{C}, i} / \Delta H_{4 \mathrm{C} \rightarrow 1 \mathrm{C} \text {, tot }}$ with variable contributions that fluctuate in a range between $19 \%$ and $45 \%$ (Fig. 8a). The variations of the mean contributions, which are $32.6 \pm 8.3 \%$ for $\Delta H_{4 \mathrm{C} \rightarrow 1 \mathrm{C}, 1}, 36.8 \pm 5.3 \%$ for $\Delta H_{4 \mathrm{C} \rightarrow 1 \mathrm{C}, 2}$, and $30.6 \pm 3.7 \%$ for $\Delta H_{4 \mathrm{C} \rightarrow 1 \mathrm{C}, 3}$, show that the heating rate has no significant effect on the overall diffusion pattern and that there is no apparent preferred path during the randomization of the vacancy distribution.

The FDSC measurements of the reversed $1 \mathrm{C}$ to $4 \mathrm{C}$ transition yield that the exothermic heat flow is characterized by an

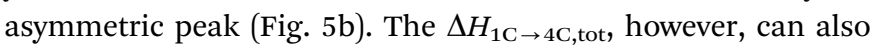
be fitted with three Gaussian components $\Delta H_{1 \mathrm{C} \rightarrow 4 \mathrm{C}, i}(i=1,2,3)$, similar to those found in the DSC measurements. The contributions of $\Delta H_{1 \mathrm{C} \rightarrow 4 \mathrm{C}, i}$ are rate-dependent. Between 10 and $30 \mathrm{~K} \mathrm{~s}^{-1}$, the average contributions of $28.8 \pm 4.6 \%$ for $\Delta H_{1 \mathrm{C} \rightarrow 4 \mathrm{C}, 1}$ is significantly lower compared to those of $38.1 \pm 3.2 \%$ for $\Delta H_{1 \mathrm{C} \rightarrow 4 \mathrm{C}, 2}$ and $33.2 \pm$ $1.5 \%$ for $\Delta H_{1 \mathrm{C} \rightarrow 4 \mathrm{C}, 3}$, respectively (Fig. 8b). At higher rates the differences between them increase drastically and the diffusion patterns split into a relative dominant $\Delta H_{1 \mathrm{C} \rightarrow 4 \mathrm{C}, 3}$, a minor $\Delta H_{1 \mathrm{C} \rightarrow 4 \mathrm{C}, 2}$, and a relatively stable $\Delta H_{1 \mathrm{C} \rightarrow 4 \mathrm{C}, 1}$. Compared to the $4 \mathrm{C}$ phase, the Coulomb repulsion between vacancies in the $1 \mathrm{C}$ structure is enhanced and the structural relation of the Fe sites and the vacancies is not explicitly defined. Given this, an unambiguous assignment of individual $\Delta H_{1 \mathrm{C} \rightarrow 4 \mathrm{C}, i}$ to specific diffusion paths is impossible. Moreover, the back-reaction to minimize the repulsion starts in a structurally undefined state and may be considered a stochastic process. This may also explain why the exothermic events cannot be isolated in the DSC and FDSC experiments.

\section{Conclusions}

The thermally-induced magneto-structural 4C to 1C transition in $\mathrm{Fe}_{7} \mathrm{~S}_{8}$ is a reversible cation-diffusion reaction in a stable hexagonal close-packing of sulfur. There is no evidence for a magnetic effect on the intrinsic diffusion processes during the transition. Three diffusion patterns during the endothermic reaction can be separated due to different migration paths and jumping frequencies of the cations. The onset of the transition is triggered by direct jumps of Fe into vacancies, destabilizing the $4 \mathrm{C}$ structure and resulting in a complex diffusion formed by dynamic variations of the vacancy distribution towards a thermally-activated state. For the back-reaction from a disordered to the ordered $4 \mathrm{C}$ structure three patterns are also identified, but they are superimposed in such a way that they cannot be explicitly resolved in the experimentally determined enthalpy changes. This probably results from the absence of a crystallographically well-defined spatial relation between Fe sites and vacancies in the $1 \mathrm{C}$ structure. Considering the experimental results, the identification of diffusion patterns based on their dynamics opens new perspectives for the investigation of intrinsic diffusive reactions in solids. This is expected to be highly beneficial for the understanding of dynamic processes in geological and technical systems that are driven by diffusion.

\section{Conflicts of interest}

There are no conflicts to declare.

\section{Acknowledgements}

The authors would like to thank Barbara Lesniak for performing the XRF measurements and Jürgen Schawe (Mettler-Toledo) for the critical discussion of the calorimetric data. The project was supported by the Swiss National Science Foundation, Grant No. 200021-153173. 


\section{References}

1 D. Koulialias, R. Schäublin, G. Kurtuldu, P. G. Weidler, J. F. Löffler and A. U. Gehring, J. Geophys. Res. Solid Earth, 2018, 123, 6236-6246.

2 C.-S. Horng and A. P. Roberts, Geochem. Geophys. Geosyst., 2018, 19, 3364-3375.

3 K. L. Louzada, S. T. Stewart and B. P. Weiss, Geophys. Res. Lett., 2007, 34, L05204.

4 C. Koike, J. Matsuno and H. Chihara, Astrophys. J., 2017, 845, 1-11.

5 D. M. Roberts, A. R. Landin, T. G. Ritter, J. D. Eaves and C. R. Stoldt, Sci. Rep., 2018, 8, 6591.

6 R. M. Hazen and R. Jeanloz, Rev. Geophys., 1984, 22, 37-46.

7 J. Zhang, Phys. Rev. Lett., 2000, 84, 507-510.

8 H.-K. Mao, J. Shu, Y. Fei, J. Hu and R. J. Hemley, Phys. Earth Planet. Inter., 1996, 96, 135-145.

9 S. A. Kissin and S. D. Scott, Econ. Geol., 1982, 77, 1739-1754.

10 P. Rochette, G. Fillion, R. Ballou, F. Brunet, B. Ouladdiaf and L. Hood, Geophys. Res. Lett., 2003, 30, 1683.

11 H. Wang and I. Salveson, Phase Transitions, 2005, 78, 547-567.

12 D. Harries, K. Pollok and F. Langenhorst, Am. Mineral., 2011, 96, 716-731.

13 C. I. Pearce, R. A. D. Pattrick and D. J. Vaughan, Rev. Mineral. Geochem., 2006, 61, 127-180.

14 K. Zhang, T. Zhang, J. Liang, Y. Zhu, N. Lin and Y. Qian, RSC $A d v .$, 2015, 5, 14828-14831.

15 M.-J. Choi, J. Kim, J.-K. Yoo, S. Yim, J. Jeon and Y. S. Jung, Small, 2018, 14, 1702816.

16 F. W. Herbert, A. Krishnamoorthy, L. Rands, K. J. Van Vliet and B. Yildiz, Phys. Chem. Chem. Phys., 2015, 17, 11036-11041.

17 E. Schwarz, Geol. Surv. Can., 1975, 1-24.

18 J. M. Rhodes, C. A. Jones, L. B. Thal and J. E. Macdonald, Chem. Mater., 2017, 29, 8521-8530.

19 C.-R. Lin, S.-Z. Lu, I. S. Lyubutin, Y. V. Korzhetskiy, S.-C. Wang and I. P. Suzdalev, J. Appl. Phys., 2010, 107, 09A335.
20 T. Takayama and H. Takagi, Appl. Phys. Lett., 2006, 88, 12512.

21 G. H. Yue, P. X. Yan, X. Y. Fan, M. X. Wang, D. M. Qu, D. Yan and J. Z. Liu, J. Appl. Phys., 2006, 100, 124313.

22 E. F. Bertaut, Acta Crystallogr., 1953, 6, 557-561.

23 D. Koulialias, E. Canévet, M. Charilaou, P. G. Weidler, J. F. Löffler and A. U. Gehring, J. Phys.: Condens. Matter, 2018, 30, 425803.

24 L. Néel, Rev. Mod. Phys., 1953, 25, 58-63.

25 E. Schwarz and D. J. Vaughan, J. Geomagn. Geoelectr., 1972, 24, 441-458.

26 U. Becker, A. W. Munz, A. R. Lennie, G. Thornton and D. J. Vaughan, Surf. Sci., 1997, 389, 66-87.

27 A. V. Powell, P. Vaqueiro, K. S. Knight, L. C. Chapon and R. D. Sánchez, Phys. Rev. B: Condens. Matter Mater. Phys., 2004, 70, 014415.

28 F. W. Herbert, A. Krishnamoorthy, B. Yildiz and K. J. Van Vliet, Appl. Phys. Lett., 2015, 106, 092402.

29 R. H. Condit, R. R. Hobbins and C. E. Birchenall, Oxid. Met., 1974, 8, 409-455.

30 G. E. Murch, J. M. Rolls and H. J. De Bruin, Philos. Mag., 1974, 29, 337-348.

31 A. A. Coelho, Bruker AXS, 2005.

32 S. Pogatscher, P. J. Uggowitzer and J. F. Löffler, Appl. Phys. Lett., 2014, 104, 251908.

33 P. Scherrer, Bestimmung der inneren Struktur und der Größe von Kolloidteilchen mittels Röntgenstrahlen, Springer, Berlin, Heidelberg, 1912, pp. 387-409.

34 J. Hopkinson, Philos. Trans. R. Soc., A, 1889, 180, 443-465.

35 O. Popov and M. Mikhov, J. Magn. Magn. Mater., 1988, 75, 135-140.

36 E. Petrovský and A. Kapička, J. Geophys. Res. Solid Earth, 2006, 111, B12S27.

37 J. L. Horwood, M. G. Townsend and A. H. Webster, J. Solid State Chem., 1976, 17, 35-42.

38 A. F. Andresen, Acta Chem. Scand., 1960, 14, 919-926.

39 H. Haraldsen, Z. Anorg. Allg. Chem., 1941, 246, 195-226.

40 H. E. Kissinger, J. Res. Natl. Bur. Stand., 1956, 57, 217-221. 